\title{
Risk of Malignant Neoplasm in Patients with Incident Rheumatoid Arthritis 1980-2007 in relation to a Comparator Cohort: A Population-Based Study
}

\author{
Shafay Raheel, ${ }^{1}$ Cynthia S. Crowson, ${ }^{2}$ Kerry Wright, ${ }^{1}$ and Eric L. Matteson ${ }^{1,2}$ \\ ${ }^{1}$ Division of Rheumatology, Mayo Clinic College of Medicine, Rochester, MN 55905, USA \\ ${ }^{2}$ Department of Health Sciences Research, Mayo Clinic College of Medicine, Rochester, MN 55905, USA \\ Correspondence should be addressed to Eric L. Matteson; matteson.eric@mayo.edu
}

Received 2 May 2016; Accepted 26 July 2016

Academic Editor: Charles J. Malemud

Copyright (C) 2016 Shafay Raheel et al. This is an open access article distributed under the Creative Commons Attribution License, which permits unrestricted use, distribution, and reproduction in any medium, provided the original work is properly cited.

Objective. To determine whether the incidence of malignancy is increased in patients with rheumatoid arthritis (RA) compared to a matched comparison cohort and to identify risk for any individual malignancy in RA. Methods. A cohort of 813 Olmsted County, Minnesota, residents who first fulfilled 1987 ACR criteria for RA in 1980-2007 was previously identified by medical record review. Medical records of 813 RA cases and a comparison cohort of age and sex matched Olmsted County residents without RA were evaluated retrospectively for cancer occurrence. Patients in both cohorts were followed until death, migration from Olmsted County, or 12/31/2014. Results. The RA and non-RA cohorts (mean age at incidence/index date: 55.9 [SD: 15.7] years; 68.4\% females in both cohorts) were followed on average of 14.1 (SD: 7.7) and 14.9 (SD: 8.1) years, respectively. Prior to RA incidence/index date, 52 RA patients and 66 non-RA subjects had malignancies excluding NMSC $(p=0.21)$. During follow-up, significantly more malignancies occurred in patients with RA $(n=143)$ than in comparator subjects $(n=118$; hazard ratio: $1.32 ; p=0.027)$. Inclusion of NMSC obviated this difference. Conclusion. After excluding NMSC, there was a small to moderately increased risk of malignancies in patients with RA. Cancer surveillance is imperative in all patients with RA.

\section{Introduction}

Patients with systemic rheumatic diseases, particularly rheumatoid arthritis (RA), systemic lupus erythematosus, systemic sclerosis, and idiopathic inflammatory myopathies, are at increased risk of developing malignancies. This risk is related to the pathobiology of the underlying rheumatic diseases including the inflammatory burden, immunological defects, and personal and environmental exposure such as smoking and some viral infections [1]. However, the occurrence of cancer among patients with RA in a nonreferral community-based population has not been thoroughly examined, especially with respect to nonmelanoma skin cancer (NMSC).

A number of studies have shown RA and RA diseases activity as pathogenic factors in the development of lymphoma $[2,3]$. Some studies have shown an increased risk of lung cancer and decreased risk of colorectal cancer in patients with RA $[4,5]$. The purpose of this study is to evaluate the occurrence of cancer both prior to and after diagnosis of RA in a population-based cohort of patients with RA and compare the occurrence to an age- and sex-matched comparison cohort without RA from the same geographical area, as well as to assess the risk factors for development of cancer among patients with RA.

\section{Materials and Methods}

2.1. Patient Cohort. This retrospective, population-based study was conducted using the resources of the Rochester Epidemiology Project, a medical records linkage system that allows ready access to the complete (inpatient and outpatient) medical records from all community medical providers [6]. An inception cohort of all cases of RA diagnosed between January 1, 1980, and December 31, 2007, among Olmsted County, Minnesota, residents $\geq 18$ years of age was previously 
assembled using the resources of the Rochester Epidemiology project [7]. Incidence date was defined as the earliest date at which the patient fulfilled at least 4 of the 7 American College of Rheumatology 1987 classification criteria for RA [8]. A comparison cohort of Olmsted County residents without RA with similar age, sex, and calendar year was also previously identified [9]. The index date for each non-RA subject was defined as the RA incidence date of the corresponding patient with RA. The institutional review boards of the Mayo Clinic and the Olmsted Medical Center approved this study.

For both the RA and comparator cohorts, cancer diagnoses were retrieved from the Mayo Clinic Cancer Registry (all malignancies except NMSC) and NMSC were abstracted from the medical charts using a standardized abstraction form. Cancer diagnoses from both before and after RA diagnosis were collected. Cancer categories included head/neck, gastric, pancreatic, liver, colon/rectal, other digestive, lung, other thorax, bone, soft tissue, skin (subdivided into melanoma and NMSC), breast, ductal carcinoma in situ, ovarian, other gynecologic, prostate, kidney, bladder, other genitourinary, ophthalmic, central nervous system, lymphoma, leukemia, multiple myeloma, myeloproliferative syndrome, myelodysplastic syndrome, and other.

The information on RA characteristics included RF status, erythrocyte sedimentation rate (ESR) at RA incidence, large joint swelling, joint erosions/destructive changes on radiographs, joint surgeries (i.e., arthroplasty and synovectomy), and extra-articular manifestations of RA (ExRA). ExRA were classified according to the criteria used in our previous studies [10]. Severe ExRA included pericarditis, pleuritis, Felty's syndrome, glomerulonephritis, vasculitis, peripheral neuropathy, scleritis, and episcleritis [11]. Data regarding start and stop dates for use of systemic glucocorticoids (e.g., oral/parental/intraarticular forms of prednisone, prednisolone, methylprednisolone, hydrocortisone, and dexamethasone), disease-modifying antirheumatic drugs (DMARDs) (methotrexate, other DMARDs), and biologic response modifiers (antitumor necrosis factor alpha [anti-TNF $\alpha$ ] agents, anakinra, abatacept, and rituximab) were collected in all patients. Smoking status was categorized as never, current, or former.

2.2. Statistical Analysis. Descriptive statistics were used to summarize data of the RA and comparator groups. The cumulative incidence of malignancy adjusted for the competing risk of death was estimated for both cohorts. This method, although similar to the Kaplan-Meier method, better accounts for patients who die before experiencing malignancy. Patients and comparator subjects with previous malignancy were excluded from analysis. These analyses were completed both as an overall estimate and by cancer type. Cox proportional hazard models were used to examine the differences between cohorts, as well as the association between patient characteristics (age, time since RA diagnosis, etc.) and the rate of development of malignancy within the RA cohort, and to assess trends in malignancy over time. Timedependent covariates were used to model risk factors that develop over time. Analyses were performed using SAS

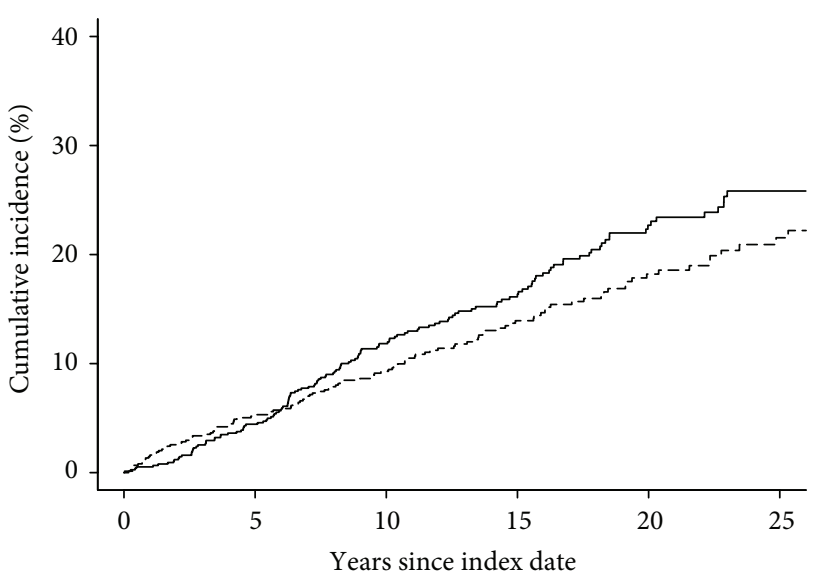

FIGURE 1: Cumulative incidence of any malignancy excluding nonmelanotic skin cancers among Olmsted County, Minnesota, residents with rheumatoid arthritis (solid line) compared to those without rheumatoid arthritis (dashed line).

version 9.4 (SAS Institute, Cary, NC, USA) and R 3.1.1 (R Foundation for Statistical Computing, Vienna, Austria).

\section{Results}

There were 813 incident cases of RA identified. The mean age of diagnosis was 55.9 (SD: 15.7) years with 42 (68.4\%) females. The mean length of follow-up after diagnosis was 14.1 (SD: 7.7) years. The majority of the patients in this study were Caucasian, $93.6 \%$ in the RA group and 94.8 in the non-RA group. In the non-RA comparator cohort the number of patients and mean age at index date were the same as for the RA cohort. The mean length of follow-up in the non-RA cohort was 14.9 (SD: 8.1 ) years. There were $21.9 \%$ and $17.7 \%$ current smokers in the RA and non-RA cohort, respectively. Prior to RA incidence/index date, 52 patients with RA and 66 nonRA subjects had malignancies excluding NMSC $(p=0.21)$. Including NMSC, there were 79 patients in the RA cohort and 108 in the non-RA cohort with malignancies ( $p=0.024)$, as there were fewer NMSC in the RA cohort than in the non-RA cohort prior to RA incidence/index date $(n=31$ versus 51 , $p=0.018)$.

3.1. Risk of Malignancy in RA Compared to Non-RA. A total of 143 patients with malignancies excluding NMSC were detected in the RA cohort, compared with 118 patients experiencing malignancies in the general population comparator cohort. The hazard ratio (HR) of any malignancy excluding NMSC was 1.32 (95\% confidence interval [CI]: 1.03, 1.68; Table 1). The cumulative incidence of any malignancy excluding NMSC at 10 years after RA incidence/index date was $11.8 \%$ (standard error [SE] 1.2\%) among the RA compared to 9.3\% (SE: 1.1\%) among the non-RA. There was no apparent difference in the cumulative incidence of any malignancy excluding NMSC during the first 5 years after RA incidence/index date (Figure 1). Including cases of NMSC, the total number of patients with malignancies in the RA cohort was 194, compared with 179 in the general population group. The HR of any 
TABLE 1: Cumulative incidence rates of malignancy in 813 patients with rheumatoid arthritis in 1980-2007 compared to 813 subjects without rheumatoid arthritis.

\begin{tabular}{|c|c|c|c|c|}
\hline Malignancy site/type ${ }^{*}$ & $\begin{array}{l}\text { Number of events } \\
\text { after incidence/index } \\
\text { in RA/non-RA }\end{array}$ & $\begin{array}{c}\text { Cumulative incidence } \\
\text { at } 10 \text { years for } \mathrm{RA} \\
\text { patients }( \pm \mathrm{SE})\end{array}$ & $\begin{array}{l}\text { Cumulative incidence } \\
\text { at } 10 \text { years for non-RA } \\
\text { subjects }( \pm \mathrm{SE})\end{array}$ & $\begin{array}{l}\text { Hazard ratio** }^{* *} \\
\quad(95 \% \mathrm{CI})\end{array}$ \\
\hline Any malignancy excluding NMSC & $143 / 118$ & $11.8 \pm 1.2$ & $9.3 \pm 1.1$ & $1.32(1.03,1.68)$ \\
\hline Any malignancy including NMSC & $194 / 179$ & $15.6 \pm 1.4$ & $14.3 \pm 1.4$ & $1.13(0.92,1.38)$ \\
\hline Any solid malignancy & $116 / 113$ & $10.3 \pm 1.1$ & $8.8 \pm 1.1$ & $1.11(0.85,1.44)$ \\
\hline Hematologic & $28 / 9$ & $1.5 \pm 0.4$ & $0.6 \pm 0.3$ & $3.58(1.69,7.60)$ \\
\hline Head/neck & $11 / 9$ & $0.9 \pm 0.3$ & $0.9 \pm 0.3$ & $1.41(0.58,3.42)$ \\
\hline Colon/rectal & $9 / 9$ & $0.8 \pm 0.3$ & $0.8 \pm 0.3$ & $1.08(0.43,2.72)$ \\
\hline Lung & $29 / 17$ & $2.1 \pm 0.5$ & $1.1 \pm 0.4$ & $1.97(1.08,3.59)$ \\
\hline Breast (among females) & $24 / 29$ & $1.8 \pm 0.6$ & $3.5 \pm 0.8$ & $0.95(0.55,1.63)$ \\
\hline Bladder & $4 / 10$ & $0.4 \pm 0.2$ & $0.6 \pm 0.3$ & $0.46(0.14,1.49)$ \\
\hline Melanoma & $11 / 13$ & $1.2 \pm 0.4$ & $0.8 \pm 0.3$ & $0.90(0.40,2.00)$ \\
\hline NMSC & $86 / 109$ & $6.3 \pm 0.9$ & $7.5 \pm 1.0$ & $0.83(0.63,1.10)$ \\
\hline Basal cell carcinoma & $57 / 75$ & $3.3 \pm 0.7$ & $5.0 \pm 0.7$ & $0.81(0.57,1.14)$ \\
\hline Squamous cell carcinoma & $56 / 69$ & $4.2 \pm 0.7$ & $4.3 \pm 0.7$ & $0.90(0.63,1.29)$ \\
\hline
\end{tabular}

NMSC = nonmelanoma skin cancer; RA = rheumatoid arthritis; $\mathrm{SE}=$ standard error; $\mathrm{CI}$ = confidence interval.

${ }^{*}$ No malignancies were observed in these sites/types: multiple myeloma, myeloproliferative syndrome, and myelodysplastic syndrome. Comparisons were not performed for the following sites/types with fewer than 5 malignancies per cohort (number of events after incidence in RA/index in non-RA): gastric (1/3), pancreatic (4/2), other digestive (2/5), other thorax (1/0), bone (1/0), soft tissue (1/4), ductal carcinoma in situ (6/4), ovary (2/1), other gynecological (4/4), kidney (2/5), other genitourinary (2/2), ophthalmologic (1/0), central nervous system (2/3), and other $(2 / 2)$.

** Adjusted for age, sex, and calendar year of RA incidence/index date. Cumulative incidence is adjusted for the competing risk of death.

malignancy including NMSC for RA compared to non-RA did not reach statistical significance (HR: 1.13; 95\% CI: 0.92, 1.38).

The incidence of hematologic cancers in patients with RA was increased compared to the general population (HR 3.58; 95\% CI: 1.69, 7.60). Of solid malignancies, lung cancer was diagnosed in 29 patients in the RA cohort; the incidence was increased compared to the non-RA cohort with HR 1.97 (95\% CI: $1.08,3.59)$.

3.2. Risk Factors for Malignancy in RA. Characteristics associated with a high risk of any malignancy included smoking (HR: 1.60; CI: 1.09, 2.34) and erosions/destructive joint changes (HR: 1.47; 95\% CI: 1.04, 2.09; Table 2). Smoking was associated with increased risk of not only lung cancer but also risk for any malignancy. The HR for any malignancy comparing current smokers to never or former smokers was 1.60 (95\% CI: 1.09, 2.34); that of lung cancer was also increased 3.59 (95\% CI: 1.23, 10.48). The risk of lung cancer in RA compared to non-RA was attenuated by adjustment for smoking status (HR: 1.48; 95\% CI: 0.81, 2.70). The risks for any malignancy (HR: 1.26; 95\% CI: 0.99, 1.62) and hematologic cancers (HR: $3.48,95 \%$ CI: 1.63, 7.41) were also somewhat smaller after adjustment for smoking status.

There were no apparent calendar time trends in the occurrence of malignancies among the RA or non-RA cohorts.

The use of glucocorticoids in patients with RA was associated with increased malignancy risk (HR 1.57; 95\% CI: $1.04,2.39)$. This association between glucocorticoid use and development of any malignancy persisted after additional adjustment for ESR at RA incidence, rheumatoid factor positivity, and current smoking (HR: 3.59; 95\% CI: 1.03, 2.40).

Methotrexate use was not associated with overall increased malignancy risk in patients with RA (HR: 1.04; 95\% CI: $0.72,1.50)$. Other DMARDs as a group were not associated with an increased risk for malignancy, although the risk of lung cancer was numerically but not significantly increased (HR: 2.38; 95\%; CI: 0.91, 6.22). As well, malignancy risk was not associated with use of biologics (primarily antitumor necrosis factor agents), glucocorticoids or antiinflammatory analgesics (Table 2). The risk of lung cancer was further attenuated by additional adjustment for erythrocyte sedimentation rate (ESR) at RA incidence, rheumatoid factor positivity, and current smoking (HR: 1.50; 95\% CI: 0.58-3.92). The malignancy risk was not increased in this RA cohort with the use of biologics (HR 0.69; CI: 0.36, 1.35).

\section{Discussion}

This retrospective population-based cohort study examined the incidence of malignancy in a nonreferral communitybased population with RA and the risk for individual malignancies, including specific solid tumors, lymphomas, leukemias, and skin cancers (NMSC and melanoma). Excluding NMSC in the risk estimation revealed an increased risk of cancer in patients with RA; however, when NMSC were included, the overall cancer risk was not increased.

In this study, the overall risk of malignancy in patients with RA was not associated with DMARDs or biologic response modifiers, principally TNF inhibitors. The use of 
TABLE 2: Risk factors for malignancy in 813 patients with rheumatoid arthritis (RA) at RA incidence and during follow-up.

\begin{tabular}{|c|c|c|c|c|}
\hline Characteristic & Value $^{* *}$ & $\begin{array}{l}\text { Hazard ratio* for any } \\
\text { malignancy }(95 \% \text { CI })\end{array}$ & $\begin{array}{c}\text { Hazard ratio* for } \\
\text { hematologic malignancy } \\
(95 \% \mathrm{CI})\end{array}$ & $\begin{array}{l}\text { Hazard ratio* for lung } \\
\text { cancer }(95 \% \mathrm{CI})\end{array}$ \\
\hline \multicolumn{5}{|l|}{ Baseline characteristics } \\
\hline Age, mean $( \pm \mathrm{SD})$ & $55.9( \pm 15.7)$ & $1.64^{\dagger}(1.44,1.86)$ & $1.98^{\dagger}(1.44,2.71)$ & $1.91^{\dagger}(1.41,2.59)$ \\
\hline Female sex & $556(68.4 \%)$ & $0.70(0.50,0.99)$ & $0.89(0.39,2.01)$ & $0.61(0.28,1.30)$ \\
\hline $\begin{array}{l}\text { Calendar year of RA incidence, } \\
\text { mean }( \pm \text { SD })\end{array}$ & $1995.6( \pm 7.9)$ & $1.06^{\dagger}(0.83,1.35)$ & $1.64^{\dagger}(0.86,3.12)$ & $1.06^{\dagger}(0.60,1.88)$ \\
\hline ESR at index, mean $( \pm S D)$ & $24.8( \pm 20.5)$ & $0.99^{\dagger}(0.90,1.09)$ & $0.89^{\dagger}(0.70,1.13)$ & $1.05^{\dagger}(0.87,1.27)$ \\
\hline $\begin{array}{l}\text { Highest ESR in 1st year, mean } \\
( \pm \mathrm{SD})\end{array}$ & $32.7( \pm 25.7)$ & $1.00^{\dagger}(0.92,1.08)$ & $0.93^{\dagger}(0.77,1.13)$ & $1.03^{\dagger}(0.88,1.21)$ \\
\hline Rheumatoid factor positive & $539(66 \%)$ & $0.91(0.64,1.30)$ & $0.79(0.35,1.77)$ & $3.59(1.23,10.48)$ \\
\hline Current smoker & $178(22 \%)$ & $1.60(1.09,2.34)$ & $0.38(0.09,1.63)$ & $22.40(8.48,59.21)$ \\
\hline Former smoker & $271(33 \%)$ & $0.91(0.63,1.31)$ & $1.60(0.72,3.56)$ & $0.42(0.17,1.06)$ \\
\hline Body mass index, $\mathrm{kg} / \mathrm{m}^{2}$ & $27.7( \pm 5.9)$ & $1.00(0.97,1.03)$ & $0.95(0.88,1.04)$ & $0.95(0.87,1.03)$ \\
\hline $\begin{array}{l}\text { Obesity (body mass index } \geq \\
30 \mathrm{~kg} / \mathrm{m}^{2} \text { ) }\end{array}$ & $244(30.0 \%)$ & $0.75(0.50,1.13)$ & $0.81(0.32,2.05)$ & $0.77(0.31,1.92)$ \\
\hline \multicolumn{5}{|l|}{ Time-dependent characteristics } \\
\hline $\mathrm{BMI} \geq 30 \mathrm{~kg} / \mathrm{m}^{2}$, ever & $391(48 \%)$ & $1.03(0.73,1.45)$ & $0.89(0.40,1.98)$ & $0.61(0.27,1.36)$ \\
\hline $\mathrm{BMI}<20 \mathrm{~kg} / \mathrm{m}^{2}$, ever & $196(24 \%)$ & $0.83(0.51,1.34)$ & $0.66(0.19,2.28)$ & $3.12(1.29,7.55)$ \\
\hline Alcohol abuse & $69(8 \%)$ & $1.82(1.07,3.10)$ & - & $7.89(3.41,18.22)$ \\
\hline Rheumatoid nodules & $272(33 \%)$ & $1.20(0.83,1.73)$ & $1.95(0.87,4.37)$ & $2.49(1.14,5.40)$ \\
\hline Erosions/destructive changes & $459(56 \%)$ & $1.47(1.04,2.09)$ & $1.88(0.81,4.34)$ & $2.65(1.14,6.16)$ \\
\hline $\begin{array}{l}\text { Severe extra-articular } \\
\text { manifestation of RA }\end{array}$ & $94(12 \%)$ & $1.41(0.84,2.35)$ & $0.79(0.19,3.38)$ & $1.99(0.75,5.29)$ \\
\hline Large joint swelling & $648(80 \%)$ & $1.06(0.70,1.60)$ & $0.85(0.33,2.17)$ & $3.42(0.81,14.54)$ \\
\hline Joint synovectomy & $89(11 \%)$ & $1.53(0.93,2.52)$ & $0.69(0.16,2.98)$ & $1.06(0.31,3.61)$ \\
\hline Joint arthroplasty & $174(21 \%)$ & $0.96(0.61,1.49)$ & $0.27(0.06,1.18)$ & $1.21(0.50,2.96)$ \\
\hline Ever 3 ESR $\geq 60 \mathrm{~mm} / 1 \mathrm{hr}$ & $114(14 \%)$ & $1.29(0.78,2.14)$ & $0.62(0.15,2.67)$ & $0.55(0.13,2.33)$ \\
\hline Methotrexate & $495(61 \%)$ & $1.04(0.72,1.50)$ & $0.74(0.31,1.73)$ & $1.49(0.66,3.39)$ \\
\hline Other DMARD & $580(71 \%)$ & $1.29(0.88,1.90)$ & $1.35(0.55,3.31)$ & $2.38(0.91,6.22)$ \\
\hline Biologics & $175(22 \%)$ & $0.69(0.36,1.35)$ & $1.16(0.32,4.14)$ & $0.34(0.04,2.61)$ \\
\hline Glucocorticoids & $650(80 \%)$ & $1.57(1.04,2.39)$ & $0.87(0.34,2.22)$ & $1.39(0.54,3.62)$ \\
\hline COX-2 inhibitor & $393(48 \%)$ & $1.09(0.74,1.60)$ & $1.52(0.65,3.55)$ & $1.10(0.47,2.58)$ \\
\hline ASA ( $\geq 1950 \mathrm{mg} /$ day; $\geq 3 \mathrm{mo}$ ) & $338(42 \%)$ & $0.76(0.51,1.13)$ & $0.53(0.21,1.34)$ & $0.63(0.26,1.52)$ \\
\hline NSAIDs for RA & $738(91 \%)$ & $0.67(0.40,1.10)$ & $1.17(0.27,5.04)$ & $0.48(0.18,1.29)$ \\
\hline
\end{tabular}

** Values are $n(\%)$ unless otherwise specified.

* Adjusted for age, sex, and calendar year of RA incidence.

${ }^{\dagger}$ Hazard ratio reported per 10 units increase.

ASA = acetylated salicylate; $\mathrm{BMI}=$ body mass index; $\mathrm{CI}=$ confidence interval; $\mathrm{ESR}=$ erythrocyte sedimentation rate; $\mathrm{COX} 2=$ cyclooxygenase 2 ; DMARD = disease modifying antirheumatic drugs; NSAID = nonsteroidal anti-inflammatory drug; RA = rheumatoid arthritis.

glucocorticoids was associated with increased risk of any malignancy, but there was no apparent association between glucocorticoids and hematologic cancers or lung cancers. The association between glucocorticoid use and development of any malignancy persisted after additional adjustment for ESR at RA incidence, rheumatoid factor positivity, and current smoking. The reason for this association is not certain. However, given the observational nature of our study, glucocorticoid use may be confounded with more severe disease and increased inflammatory burden, which may be associated with malignancy risk.
The overall increased risk of cancer was largely driven by the increased risk of hematologic cancers. A link between lymphoma and RA was first reported from a medical record linkage study in 1978 [12]. Subsequently, a considerable body of evidence has emerged that supports RA and RA disease activity as pathogenic factors in the development of lymphoma $[2,3]$. In a meta-analysis of 21 publications from 1990 to 2007 on the risk of malignancy in patients with RA, the risk of lymphoma was increased approximately twofold (SIR 2.08, 95\% CI 1.8, 2.39), with a greater risk of both Hodgkin's and non-Hodgkin's lymphoma [13]. Lymphoproliferative 
malignancies are also increased in patients with extraarticular disease manifestations such as Felty's syndrome [14] and secondary Sjögren's syndrome [15].

Pooled data from 74 randomized controlled trials showed that TNF inhibitors were associated with an increase in risk of NMSC beyond the risk associated with RA alone [16]. Several large observational studies have supported this finding [17, 18], but others have not [19-21]. In a recent population-based cohort study conducted in Sweden, the risk of squamous cell cancer (SCC) and basal cell cancer (BCC) was evaluated in patients with RA naïve to biologic drugs, in patients starting TNF inhibitors treatment, and in the general population. This study demonstrated a $20 \%$ increased risk of BCC and a near doubled risk of SCC in patients with RA compared with the general population. For patients treated with TNF inhibitors compared with those naïve to biologics, BCC risk was moderately increased, but the increase was not significant after adjustments for demographic and comorbidity variables. Limitations of that study include that the authors were unable to adjust for severity of disease. People with more severe arthritis could be more likely to receive TNF inhibitor. If severity of arthritis is related to risk of NMSC, then this outcome is confounded by indication. Patients were not randomly assigned to treatments in this observational study, so any excess risk could be due to increased severity of disease rather than treatment [22].

One of the studies conducted among 13,001 patients using the US National Cancer Institute SEER (Surveillance, Epidemiology, and End Results) database revealed increased risk for skin cancers with biologic therapy, but not for solid tumors or lymphoproliferative malignancies. These associations were consistent across different biologic therapies [17]. Another study from The South Swedish Arthritis Treatment Group register (SSATG) evaluated the risk of malignancy in patients who had undergone TNF inhibitor therapy. This study demonstrated that patients receiving conventional RA treatment had an increased overall tumor risk compared with the background population. Possible additional increased risk for lymphoma associated with TNF inhibitors was also reported in few cases [23].

The risk for lung cancer was increased in the current study. An increased risk of lung cancer has been reported in individual studies [4], as well as in the meta-analyses [13]. This may be related to an increased risk of RA in smokers described in population-based prospective cohort studies $[24,25]$. Conversely, in a study of patients with RA in the US veterans' population, the risk of lung cancer was increased by $43 \%$ compared with the general population, even after adjustment for tobacco and asbestos exposure [26].

Strengths of this study include its population-based design and complete medical record review. The Rochester Epidemiology project affords the ability to include both patients with RA and age- and sex-matched comparator subjects living within the same community, reducing biases of referral populations. The average follow-up of 14 years in this study is much longer than the majority of other retrospective studies [13]. The length of follow-up in the current study permits assessment of long-term risk and secular trends in cancer development compared to most studies which are of shorter duration.

Limitations may include the fact that the population of Olmsted County is predominately Caucasian; however results of REP studies are generally applicable to other population cohorts [26]. In addition, there is some concern with the size of our cohort, 813 RA patients, 7800 patient-years of followup. While our study was adequately sized to detect increased risk for any malignancy, it was under-powered to detect increased risks for cancer subtypes.

\section{Conclusion}

There was a small to moderately increased risk of malignancies excluding NMSC in RA patients; the risk was highest for hematologic cancers. Risk for lung cancer was also increased. The overall risk of malignancy in patients with RA was not associated with DMARDs or biologic response modifiers, principally TNF inhibitors. Cancer surveillance is imperative in all patients with RA.

\section{Disclosure}

The content is solely the responsibility of the authors and does not necessarily represent the official views of the National Institutes of Health.

\section{Competing Interests}

The authors declare that they have no competing interests.

\section{Acknowledgments}

This work was funded by a grant from the National Institutes of Health, NIAMS (R01 AR46849). Research reported in this paper was supported by the National Institute on Aging of the National Institutes of Health under Award no. R01AG034676.

\section{References}

[1] C. Turesson and E. L. Matteson, "Malignancy as a comorbidity in rheumatic diseases," Rheumatology, vol. 52, no. 1, pp. 5-14, 2013.

[2] L. Mellemkjær, M. S. Linet, G. Gridley, M. Frisch, H. Møller, and J. H. Olsen, "Rheumatoid arthritis and cancer risk," European Journal of Cancer Part A, vol. 32, no. 10, pp. 1753-1757, 1996.

[3] F. Wolfe and K. Michaud, "Lymphoma in rheumatoid arthritis: the effect of methotrexate and anti-tumor necrosis factor therapy in 18,572 patients," Arthritis and Rheumatism, vol. 50, no. 6, pp. 1740-1751, 2004.

[4] M. Kauppi, E. Pukkala, and H. Isomäki, "Excess risk of lung cancer in patients with rheumatoid arthritis," The Journal of Rheumatology, vol. 23, no. 8, pp. 1484-1485, 1996.

[5] M. Kauppi, E. Pukkala, and H. Isomäki, "Low incidence of colorectal cancer in patients with rheumatoid arthritis," Clinical and Experimental Rheumatology, vol. 14, no. 5, pp. 551-553, 1996.

[6] J. L. St. Sauver, B. R. Grossardt, B. P. Yawn, L. J. Melton III, and W. A. Rocca, "Use of a medical records linkage system to enumerate a dynamic population over time: the Rochester Epidemiology Project," American Journal of Epidemiology, vol. 173, no. 9, pp. 1059-1068, 2011. 
[7] E. Myasoedova, C. S. Crowson, H. M. Kremers, T. M. Therneau, and S. E. Gabriel, "Is the incidence of rheumatoid arthritis rising? Results from Olmsted County, Minnesota, 1955-2007," Arthritis and Rheumatism, vol. 62, no. 6, pp. 1576-1582, 2010.

[8] F. C. Arnett, S. M. Edworthy, D. A. Bloch et al., "The American Rheumatism Association 1987 revised criteria for the classification of rheumatoid arthritis," Arthritis \& Rheumatism, vol. 31, no. 3, pp. 315-324, 1988.

[9] A. K. Bacani, S. E. Gabriel, C. S. Crowson, J. A. Heit, and E. L. Matteson, "Noncardiac vascular disease in rheumatoid arthritis: increase in venous thromboembolic events?" Arthritis and Rheumatism, vol. 64, no. 1, pp. 53-61, 2012.

[10] C. Turesson, W. M. O’Fallon, C. S. Crowson, S. E. Gabriel, and E. L. Matteson, "Extra-articular disease manifestations in rheumatoid arthritis: incidence trends and risk factors over 46 years," Annals of the Rheumatic Diseases, vol. 62, no. 8, pp. 722-727, 2003.

[11] C. Turesson, L. Jacobsson, and U. Bergström, "Extra-articular rheumatoid arthritis: prevalence and mortality," Rheumatology, vol. 38, no. 7, pp. 668-674, 1999.

[12] H. A. Isomäki, T. Hakulinen, and U. Joutsenlahti, "Excess risk of lymphomas, leukemia and myeloma in patients with rheumatoid arthritis," Journal of Chronic Diseases, vol. 31, no. 11, pp. 691-696, 1978.

[13] A. L. Smitten, T. A. Simon, M. C. Hochberg, and S. Suissa, "A meta-analysis of the incidence of malignancy in adult patients with rheumatoid arthritis," Arthritis Research \& Therapy, vol. 10, no. 2, article R45, 2008.

[14] G. Gridley, J. H. Klippel, R. N. Hoover, and J. F. Fraumeni Jr., "Incidence of cancer among men with the Felty syndrome," Annals of Internal Medicine, vol. 120, no. 1, pp. 35-39, 1994.

[15] S. S. Kassan, T. L. Thomas, H. M. Moutsopoulos et al., "Increased risk of lymphoma in sicca syndrome," Annals of Internal Medicine, vol. 89, no. 6, pp. 888-892, 1978.

[16] J. Askling, K. Fahrbach, B. Nordstrom, S. Ross, C. H. Schmid, and D. Symmons, "Cancer risk with tumor necrosis factor alpha (TNF) inhibitors: meta-analysis of randomized controlled trials of adalimumab, etanercept, and infliximab using patient level data," Pharmacoepidemiology and Drug Safety, vol. 20, no. 2, pp. 119-130, 2011.

[17] W. Amari, A. L. Zeringue, J. R. McDonald, L. Caplan, S. A. Eisen, and P. Ranganathan, "Risk of non-melanoma skin cancer in a national cohort of veterans with rheumatoid arthritis.," Rheumatology (Oxford, England), vol. 50, no. 8, pp. 1431-1439, 2011.

[18] L. K. Mercer, A. C. Green, J. B. Galloway et al., "The influence of anti-TNF therapy upon incidence of keratinocyte skin cancer in patients with rheumatoid arthritis: longitudinal results from the British Society for Rheumatology Biologics Register," Annals of the Rheumatic Diseases, vol. 71, no. 6, pp. 869-874, 2012.

[19] L. Dreyer, L. Mellemkjær, A. R. Andersen et al., "Incidences of overall and site specific cancers in TNF $\alpha$ inhibitor treated patients with rheumatoid arthritis and other arthritides-a follow-up study from the DANBIO Registry," Annals of the Rheumatic Diseases, vol. 72, no. 1, pp. 79-82, 2013.

[20] K. Haynes, T. Beukelman, J. R. Curtis et al., "Tumor necrosis factor $\alpha$ inhibitor therapy and cancer risk in chronic immunemediated diseases," Arthritis and Rheumatism, vol. 65, no. 1, pp. 48-58, 2013.

[21] P. Raaschou, J. F. Simard, C. A. Hagelberg, J. Askling, and ARTIS Study Group, "Rheumatoid arthritis, anti-tumour necrosis factor treatment, and risk of squamous cell and basal cell skin cancer: cohort study based on nationwide prospectively recorded data from Sweden," The British Medical Journal, vol. 352, article i262, 2016.

[22] S. Assassi, "Rheumatoid arthritis, TNF inhibitors and nonmelanoma skin cancer," The British Medical Journal, vol. 342, article i472, 2016.

[23] P. Geborek, A. Bladström, C. Turesson et al., "Tumour necrosis factor blockers do not increase overall tumour risk in patients with rheumatoid arthritis, but may be associated with an increased risk of lymphomas," Annals of the Rheumatic Diseases, vol. 64, no. 5, pp. 699-703, 2005.

[24] M. Heliovaara, K. Aho, A. Aromaa, P. Knekt, and A. Reunanen, "Smoking and risk of rheumatoid arthritis," Journal of Rheumatology, vol. 20, no. 11, pp. 1830-1835, 1993.

[25] U. Bergström, L. T. H. Jacobsson, J.-Å. Nilsson, G. Berglund, and C. Turesson, "Pulmonary dysfunction, smoking, socioeconomic status and the risk of developing rheumatoid arthritis," Rheumatology, vol. 50, no. 11, pp. 2005-2013, 2011.

[26] R. Khurana, R. Wolf, S. Berney, G. Caldito, S. Hayat, and S. M. Berney, "Risk of development of lung cancer is increased in patients with rheumatoid arthritis: a large case control study in US veterans," Journal of Rheumatology, vol. 35, no. 9, pp. 17041708, 2008. 


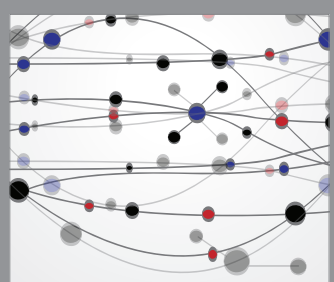

The Scientific World Journal
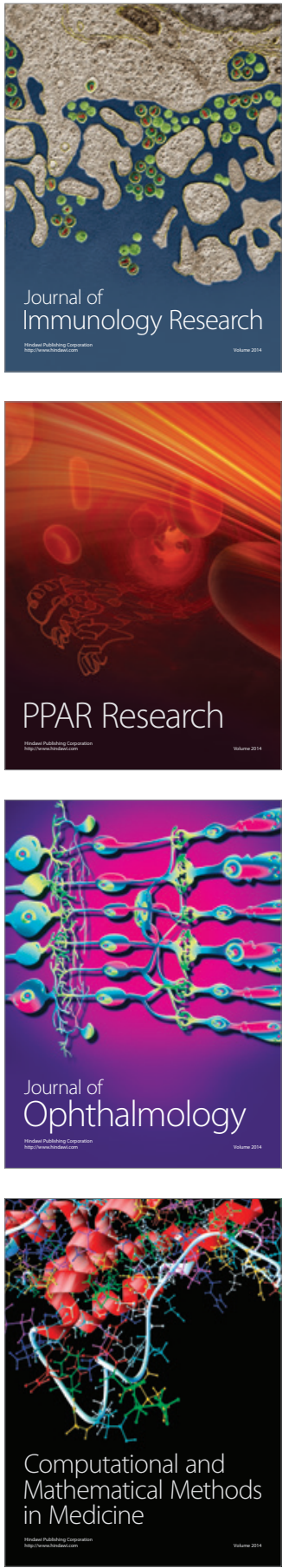

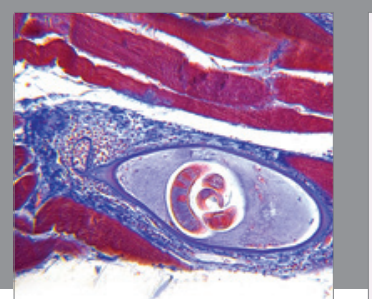

Gastroenterology Research and Practice

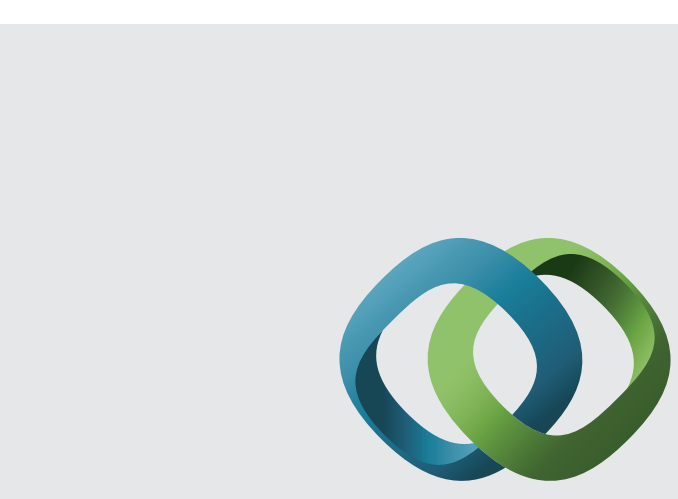

\section{Hindawi}

Submit your manuscripts at

http://www.hindawi.com
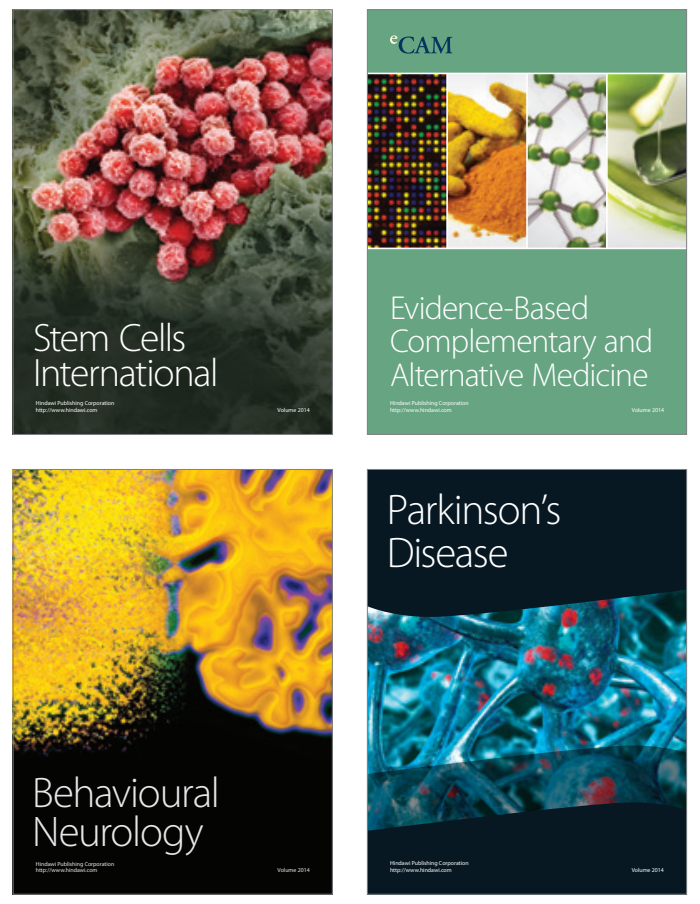
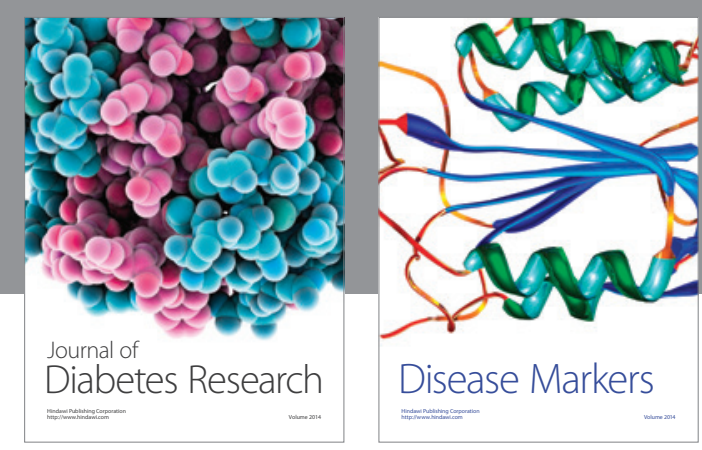

Disease Markers
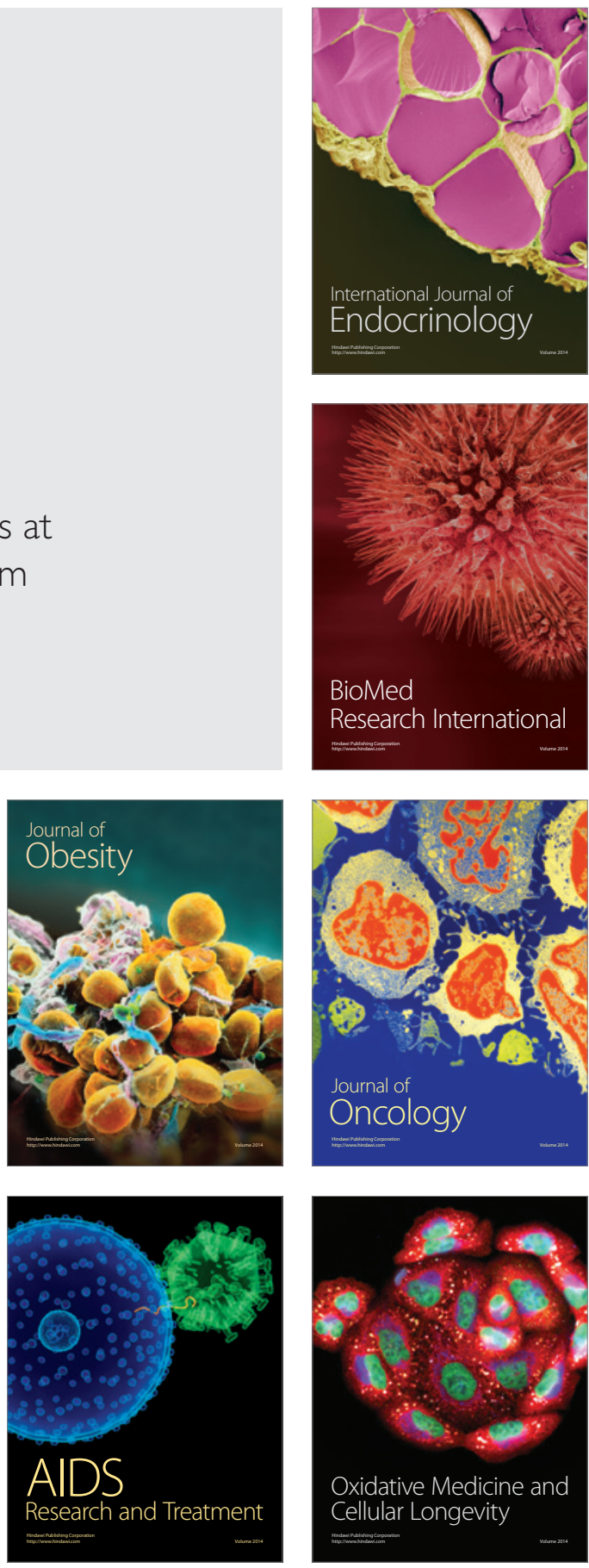